\title{
A Comparative Study of the Effectiveness of Drug Addiction Treatment Methods Used at the Drug Addiction Treatment Center of Jahrom in 2014
}

\author{
Razieh Parniyan ${ }^{1}$, Abdoreza Kazemiane ${ }^{2}$, \\ Marzieh Kargar Jahromi ${ }^{3}$ and Farzad Poorgholami ${ }^{4}$ \\ ${ }^{1}$ Department of Nursing, Faculty of Nursing and Midwifery, \\ Jahrom University of Medical Sciences, Jahrom, Iran. \\ ${ }^{2}$ Practitioner of Medical University, Jahrom University of Medical Sciences, Jahrom, Iran. \\ ${ }^{3}$ Community Health Nursing, Jahrom University of Medical Science, Jahrom, Iran. \\ ${ }^{4}$ Medical-Surgical Nursing, Jahrom University of Medical Science, Jahrom, Iran. \\ http://dx.doi.org/10.13005/bbra/2030
}

(Received: 19 January 2016; accepted: 27 February 2016)

\begin{abstract}
In recent years, a lot of efforts have been made toward developing new methods for treating drug addicts. The present study aims to compare the effectiveness of the drug addiction treatment methods used for the addicts at the drug addiction treatment center of Jahrom, Iran in 2014. The present study is a retrospective cohort study whose population consisted of all the individuals who were undergoing treatment at the medical center of MMT in Jahrom. Sampling of the files of the entire patients (150) was based on the census method. The researchers studied each treatment group separately. The data collected from the patients' files were included their personal and medical information and level of improvement. The results of the patients' treatment plans (MMT, BMT, and opium tincture) were collected, compared and analyzed. For the statistical analysis of the data, the researchers used descriptive indexes (frequency and percentage), the chi-square test, Pearson's correlation coefficient, and SPSS v. 24. Based on the collected data, the effectiveness of the treatments was as follows: weak $(30 \%)$ in $6.0 \%$ of the BMT cases and $\mathbf{1 2 . 0 \%}$ of MMT cases; moderate (50\%) in $\mathbf{7 2 . 0} \%$ of the BMT cases and $27.8 \%$ of MMT cases; and satisfactory $(60 \%)$ and above in $22.0 \%$ of the BMT cases and $60.3 \%$ of MMT cases, all of which values were statistically significant. The results of the study show that using methadone is a more effective method for treating drug abuse compared to other treatments.
\end{abstract}

Keywords: treatment, drug addiction, drug addicts, drug treatment center.

Drug abuse and addiction is a multifaceted universal issue believed to be caused by a variety of factors, among them social, economic, personal and environmental factors. Studies show that the number of individuals addicted to illegal drugs is on the rise across the world, and Iran is no exception. According to the ministry of health of Iran and the drug abuse treatment protocol, 200

\footnotetext{
* To whom all correspondence should be addressed. Tel.: 98-71-5434-1501;

E-mail: FarzadPoorgholami1393@gmail.com
}

million people in the world are addicted to substances other than alcohol, 162 million of whom are addicted to the less harmful hashish. In Iran, however, opium and heroin addiction prevail ${ }^{1}$.

Presently, there are approximately 3 million drug addicts in Iran, including casual drug users. Studies show that the majority of the more than 1 million and 2 hundred thousand of the individuals addicted to illegal drugs in Iran are dependent on synthetic drugs rather than traditional drugs, and heroin addiction poses the most serious threats to addicts and the society: compared to opium addicts, heroin addicts are much less capable of quitting 
permanently and returning to a normal life ${ }^{2}$.

Statistics show that the rate of deaths caused by drug abuse, especially overdose, is increasing is Iran. According to one study, if the cost of heroin used by an addict is 5000 tomans a day and supposing that there are 250 thousand heroin addicts in Iran, the total cost of heroin used by these addicts will come to 37.5 billion tomans a month and 450 billion tomans a year ${ }^{3}$.

The worst type of addiction in Iran is associated with drugs that are administered intravenously. Unfortunately, drug abuse by villagers is on the increase and an increasing number of younger individuals and females are becoming addicted to illegal drugs. According to the chief of the Welfare Institute of Iran, the growth rate of drug abuse (8\%) is three times as fast as the birth rate (2.6\%). Moreover, many divorces are attributed to drug abuse and official statistics show that at least 6 million individuals in Iran suffer directly or indirectly as a result of drug abuse by a second party ${ }^{4,5}$.

According to WHO, drug abuse has the three following characteristics:

1. A tendency and need for obtaining and using the drug in question in any possible way,

2. An inclination to use a larger amount of the drug in question every day,

3. Physical and mental disorders caused by drug abuse $\mathrm{e}^{6,7}$.

Obviously, the first and most important step toward preventing social harms is fighting drug abuse; after all, prevention is better than cure. Despite the many efforts made by researchers and various international organizations, a fully effective method for treating drug abuse has not been discovered yet $(2,4)$. Fortunately, in recent years, there has been an increase in the acceptance of the belief that drug addiction is a disease, not a crime, and a variety of approaches have been employed to treat drug addicts ${ }^{8}$.

Treating drug abuse is a complicated process: on the most obvious level, this is a twodimensional disease, i.e. it involves both physical and psychological dependence. A treatment plan that fails to consider either of these factors is an incomplete and reversible cure. The efforts that have been made in the domains of medicine and psychology toward finding the most effective method of treatment are quite promising; however, due to individual difference between addicts and their reasons for drug abuse and the occasional launch of designer drugs, it is necessary that new methods of treatment be developed and employed. With the spread of drug abuse treatment programs under various names, each clinical system claims to offer effective plans with unique features ${ }^{9,10}$. Among such institutes are the drug addiction treatment clinics that are affiliated to the Ministry of Health of Iran and aim to attract and help drug addicts by offering them new methods of treatment.

The most popular drugs used in Iran for the purpose of maintenance therapy are methadone, buprenorphine, and opium tincture. Among the features of these drugs that make them good choices are: they are administered orally and there is no need for injection; they are pure and do not contain secondary chemicals with unknown effects; they have long-lasting effects and do not need to be taken frequently; they have a clearer absorption and excretion cycle compared to opium and heroin and can, therefore, be administered safely with a low risk of toxicity ${ }^{11}$.

Moreover, since the above-mentioned dugs can be acquired at clinical centers under a doctor's supervision, they reduce drug addicts' dependence on drug dealers and consequently decrease the social, penal, and economic costs associated with illegal drugs. Replacing illegal drugs with legal medical chemicals will cut drug dealers' profits and hinder the expansion of drug sales and trafficking. Also, as these medical drugs are heavily subsidized and are available at low costs, drug addicts will not need to rely on antisocial activities to make enough money to buy drugs. Overall, the above-mentioned advantages of maintenance therapies make them the corner stones of drug abuse treatment in developed countries $^{12,13}$.

A review of the available studies reveals that, despite attempts at comparing the effectiveness of various methods of treatment, it is not clear which method(s) is the optimum choice. Moreover, the present study is the first attempt at comparing the impact of the methods of treatment currently used at the Drug Addiction Treatment Center of Jahrom, Iran on improving the conditions of drug addicts and determining the best method. 


\section{METHOD}

\section{Type of study}

The present study is a retrospective cohort study (file review).

\section{Population}

The entire patients who were undergoing treatment at the MMT Clinical Center of Jahrom, Iran.

\section{Sampling}

The sample was selected from the entire patients' files (150) at the MMT Clinical Center using the census method. The patients in each

Table 1. The demographic characteristics of the subjects

\begin{tabular}{lcc}
\hline Variable & Group & Percentage \\
\hline \multirow{4}{*}{ Age (years) } & Under 20 & 3.47 \\
& $21-30$ & 26.25 \\
& $31-40$ & 34.36 \\
Gender & $41-50$ & 23.93 \\
& Above 50 & 11.96 \\
Marital status & Male & 95.83 \\
& Female & 4.47 \\
& Single & 47.29 \\
Education & Married & 50.39 \\
& Divorced & 2.32 \\
& Junior high school & 79.54 \\
Employment & High school & 16.99 \\
& Post high school & 1.54 \\
& BA/BS & 1.93 \\
Income & Jobless & 11.81 \\
& State employee & 4.72 \\
& Self-employed & 83.07 \\
& Retired & 0.394 \\
& Low & 37.45 \\
& Average & 49.42 \\
& Good & 13.13 \\
\hline
\end{tabular}

treatment group-BMT, MMT, and opium tincture-were selected for study based on their number in their group.

\section{A summary of the implementation process of the project}

After the study project had been approved at Jahrom University of Medical Sciences, the required permit was submitted to the treatment center and the files of all the patients who had completed their treatment plans were examined. At the center in question, method of treatment for each patient was determined thus: based on the type and amount of the substance used, method of administration, length of addiction, the number of attempts made to quit in the past and the doctor's judgment, one of the three methods of MMT, BMT, or opium tincture would be prescribed for each addict. Regardless of the type of treatment prescribed, the treatment procedure is as follows: 1 . Induction (the initial administration of a certain amount of the prescribed drug based on the patient's symptoms and history); 2. Stability (fixing the amount of the drug administered based on the patient's withdrawal symptoms); 3. Maintenance (regular administration of the prescribed drug); 4. Tapering (gradually decreasing the amount administered).

The inclusion criterion was having been a patient at MMT Drug Addiction Treatment Center, and the exclusion criterion was not having completed one's treatment program. In the present study, the researchers studied the subjects in each treatment group separately. The data extracted from the patients' files included personal and medical information and level of improvement. To evaluate the patients' level of improvement, the researchers used medical and psychological checklists, their laboratory test results, and their doctors' comments

Table 2. A comparison between the effectiveness levels of the three methods of drug abuse treatment (MMT, BMT and opium tincture) as used at the MMT Drug Addiction Treatment Center of Jahrom, Iran

\begin{tabular}{|c|c|c|c|c|c|c|c|c|}
\hline \multirow[t]{2}{*}{$\begin{array}{l}\text { Recovery } \\
\text { Method }\end{array}$} & \multicolumn{2}{|c|}{$\begin{array}{c}\text { Poor } \\
\text { Below } 30 \%\end{array}$} & \multicolumn{2}{|c|}{$\begin{array}{l}\text { Moderate } \\
30 \% \text { to } 50 \%\end{array}$} & \multicolumn{2}{|c|}{$\begin{array}{c}\text { Satisfactory } \\
\text { Above 50\% }\end{array}$} & \multirow[b]{2}{*}{ Static } & \multirow[b]{2}{*}{ P-value } \\
\hline & $\mathrm{F}$ & $\mathrm{P}$ & $\mathrm{F}$ & $\mathrm{P}$ & $\mathrm{F}$ & $\mathrm{P}$ & & \\
\hline MMT & 25 & $12.0 \%$ & 58 & $27.8 \%$ & 126 & $60.3 \%$ & & \\
\hline Tincture & 1 & $25.0 \%$ & 1 & $25.0 \%$ & 2 & $50.0 \%$ & 35.22 & $\mathrm{P} \leq 0.001$ \\
\hline $\mathrm{BMT}$ & 3 & $6.0 \%$ & 36 & $72.0 \%$ & 11 & $22.0 \%$ & & \\
\hline Total & 29 & $11.0 \%$ & 95 & $36.1 \%$ & 139 & $52.9 \%$ & & \\
\hline
\end{tabular}


as recorded in the patients' files.

To describe each patient's improvement status, the researchers used the two options of "recovered" and "unrecovered." The recovered patients were those who tested negative for illegal drugs, did not demonstrate addict-like behaviors, had mended their ways and reassumed their personal, family and social roles and, overall, were judged to be in good physical and mental health.

\section{Data analysis}

The data collected from each treatment group-MMT, BMT, and opium tincture-were compared and analyzed using descriptive indexes (frequency and percentage), the chi-square test, and Pearson's correlation coefficient in SPSS v. 24.

\section{RESULTS}

Table 1 shows the subjects' demographic characteristics. The results of the chi-square test (Table 2) show that there are significant differences between the three methods of BMT, MMT, and opium tincture in terms of the level of recovery each brings about. Also, based on the collected data, the effectiveness of the treatments was as follows: weak (30\%) in $6.0 \%$ of the BMT patients and $12.0 \%$ of MMT patients; moderate (50\%) in $72.0 \%$ of the BMT patients and $27.8 \%$ of MMT patients; and satisfactory and above (60\%) in $22.0 \%$ of the BMT patients and $60.3 \%$ of MMT patients, all of which values were statistically significant.

\section{DISCUSSION}

In the present study, satisfactory (and above) levels of recovery were found to have been achieved by $60.3 \%$ of the patients who had been administered methadone, $22 \%$ of the patients who had been administered buprenorphine, and 50\% of the patients who had been administered opium tincture.

Since 1964, methadone maintenance therapy (MMT) has been widely recommended based on the assumption that high doses of methadone can eliminate one's dependence on illegal drugs and, by creating a parallel source of satisfaction, prevents withdrawal symptoms. Thus, the users of addictive drugs will be freed from their mental occupation with drugs and desperate efforts to achieve drugs and can direct their energies toward more productive highways, ${ }^{6,14}$.

The results of the present study are consistent with the findings of the study of Torrens et al. who report that MMT reduces social alienation in addicts and improves their sleep quality, which means that it reduces depression in addicts. Likewise, Giacomuzzi et al. (2002) report that methadone improves drug addicts' physical conditions: MMT enhances energy levels and physical performance and reduces pain in drug addicts. Torrens et al. report similar findings. Evidently, MMT can not only reduce or eliminate one's dependence on drugs, but improves psychological health and lowers the rate of social crimes $^{21,22}$.

On the other hand, administration of buprenorphine is a much less popular treatment in Iranian clinics: currently, out of the 500,000 drug addicts who are undergoing maintenance therapy in Iran, 430,000 (87\%) are on methadone and 65,000 (13\%) are on buprenorphine ${ }^{15}$. Buprenorphine (Buprexin) is a partial agonist, meaning that, though it is an addictive substance and can cause the common effects and side effects associated with other addictive drugs, such as shortness of breath and ecstasy, its maximum influence is weaker than the influence of such full agonists as heroin and methadone. In small doses, buprenorphine can generate enough agonistic effects to enable a drug addict to quit drugs without experiencing the withdrawal symptoms.

The agonistic effects of buprenorphine (Buprexin) grow in a linear manner as doses increase until they reach a plateau; at that point, an increase in dose will not lead to stronger effects ${ }^{16}$. This condition is called the ceiling effect. Accordingly, compared to full agonists, the use of buprenorphine is less likely to result in abuse, addiction and side effects. In fact, buprenorphine can block the effects of full agonists and consequently cause serious withdrawal symptoms in a drug addict. This is because of the high tendency of buprenorphine to cling to drug receptors: buprenorphine has a greater tendency to bind to drug receptors than other drugs do, which property helps it defeat other drugs in attaching to these receptors ${ }^{17}$.

In the present study, only 50 of the 263 patients who visited the clinic were found to use buprenorphine. Some of the reasons why 
buprenorphine is not administered very often in drug addiction treatment centers in Iran are as follows: variety in the brands of buprenorphine, patients and therapists' reluctance to choose buprenorphine, the high costs and low profits of the administration of buprenorphine at drug addiction treatment centers, the side effects of buprenorphine and therapists' lack of knowledge about how to deal with the side effects ${ }^{18}$. For new methods of treatment to become popular, therapists should accept that there are other drugs than methadone.

According to Dr. Daneshmand, therapists' choice of drug is partly influenced by the costs and medical subsidies; he states that the cost of MMT is 1,164,000 rials per month, while the cost of BMT (with $10 \mathrm{mg}$ tablets on average) is $1,326,000$ rials, and a non-medication treatment plan can cost 621,000 rials. He believes that with efficient management, more patients can be put on buprenorphine and the profits of clinics can be increased $^{19}$.

In the present study, the moderate recovery level achieved by buprenorphine was found to be twice as much as methadone (70\% versus $30 \%$ ). The researchers recommend that medical universities and the ministry of health choose the better-known brands of buprenorphine and improve its distribution process to encourage therapists and patients to select buprenorphine for treatment. Buprenorphine often causes only a small number of side effects (its most common side effect is headaches) which can be easily controlled if a patient cannot tolerate them. Recent studies recommend buprenorphine over methadone as the number one drug for treating drug addicts. If a patient with withdrawal symptoms and a serious concurrent disease desires a long-term recovery program, maintenance therapy with legal agonists should be suggested to him or her immediately. The fact that maintenance therapy with legal agonists is more effective than detoxification methods in reducing the rates of side effects and deaths has been proven beyond doubt ${ }^{17,20,22}$.

Comparing the effectiveness of two detoxification methods for opium-dependent patients, Taraghi et al. (2005) discovered that methadone and buprenorphine acted similarly in controlling the patients' withdrawal symptoms; yet, the patients were more willing to use methadone because of its lower cost.

\section{CONCLUSION}

The findings of the study show that, compared to the other methods of treatment under study, the administration of methadone can make a greater contribution to the recovery of drug addicts and is recommended as an effective method.

\section{REFERENCES}

1. Beigi, Ali. The Diagnostic Role of Quality of Life and Hope in Anonymous Addicts and Addicts undergoing Methadone Maintenance Therapy. The Journal of Clinical psychology. Year 3, No. 3 issue 11. Fall 2011.

2. Khayatipour, Atefeh. Comparing the Effectiveness of Combined Cognitive-behavioral Therapy in Community Therapy and Narcotic Anonymous Groups on Their Tendency to Abuse Drugs. The Journal of Science and Medicine. 9(6). 2010.

3. Yarmohammadi Vasel, Mosayeb. The Effects of Therapeutic Community on Improving Male Drug Addicts’ Life Skills and Reducing Their Tendency to Relapse. The Journal of Kerman University of Medical Sciences. 18(4). 2011. pp. 358-368.

4. A Comparison of the Effects of the Oral Administration of Harmal Seeds and Methadone on the Behavioral Indexes of Male Rats' Withdrawal Symptoms. Modares Journal of Medical Sciences: Pathobiology. 2010; 13(1). pp. 37-46.

5. Akbari, Aliasghar; Peiman Ghadirjahrom. The Effects of Alcoholic Drinks, Tea and Coffee from a Medical Viewpoint.

6. Kaplan H.I. MD, Saddock B.J. MD, Grebb J.A. MD. Comprehensive textbook of psychiatry. $7^{\text {th }}$ ed. William and Wilkins; 1999.1055-57.

7. A Clinical Guide to Treating Poisoning with Stimulants. The Ministry of Health and Medical Education. The Office of Mental-social Health and Drug Abuse. Sepidbarg Publishing Company. 2011. Tehran.

8. Norouzi, Alireza. The Principles of Treating Substance Dependence. The Ministry of Health and Medical Education. The Office of Mentalsocial Health and Drug Abuse. Sepidbarg Publishing Company. 2011. Tehran 
9. Sadeghi, Maneli. (Trans.) A Guide to Treating Methamphetamine Abuse. Sepidbarg Publishing Company. 2011. Tehran.

10. Momtazi, Saeed. The Science of Addiction: Drug Abuse, the Brain and Behavior. The Ministry of Health and Medical Education. The Office of Mental-social Health and Drug Abuse. Sepidbarg Publishing Company. 2011. Tehran.

11. Taheri, Hammidreza. A Guide Book to Psychological Interventions for Outpatients Addicted to Stimulants based on the Matrix Model. The Ministry of Health and Medical Education. The Office of Mental-social Health and Drug Abuse. Sepidbarg Publishing Company. 2012. Tehran.

12. Norouzi, Alireza. A Guide to Drug Abuse Treatment of Inpatients. The Ministry of Health and Medical Education. The Office of Mentalsocial Health and Drug Abuse. Sepidbarg Publishing Company. 2011. Tehran.

13. Soltani, Hadieh. (Trans.) Marijuana: Facts for Parents and Adolescents. The National Institute of Drug Abuse. Sepidbarg Publishing Company. 2012. Tehran.

14. Ahmadpanah M, Mirzaei Alavijeh M, AllahverDipour H, Jalilian F, Haghighi M, Afsar A, GhaRibnavaz H. Effectiveness of coping skills education program to reduce craving beliefs among addicts referred to addiction centers in Hamadan: a randomized controlled trial. Iranian J Publ Health 2013; 42(10): 1139-44.

15. Jalilian F, Allahverdipour H, Moinei BPh, Moghimbeigi A, Barati M, MirzaeiAlavijeh M. Prevention of Anabolic Steroids use among Gym users: Application of Peer Education Approach. The Journal of Toloo-e-behdasht 2011; 10(2): 45-56. [In Persian]
16. Poorgholami F, Abdollahifard F, Zamani M, KargarJahromi M, BadiyepeymaJahromi Z. The Effect of Stress Management Training on Hope in Hemodialysis Patients. Global Journal of Health Science. 2016; 8(7).165-171

17. Poorgholami F, Mansoori P, Montaseri Z, Najafi K. Effect of Self-Care Education with and without Telephone Follow-Up on the Level of Hope in Hemodialysis Patients; A Randomized Clinical Trial. IJCBNM. 2016; 4(2):90-97.

18. Sabetgadam M, Poorgholami F, BadiyepeymaieJahromi F, Parandavar F, Kalani N, Rahmanian E. Effect of Self-Care Education by Face-to-Face Method on the Quality of Life in Hemodialysis Patients. Global Journal of Health Science. 2016; 8(6):140-147.

19. Poorgholami F, RahmanianKoshkaki A, KargarJahromi M, Parniyan R. A Study of the Influence of Group-Based Learning of Stress Management on Psychology Symptoms Levels of Hemodialysis Patients. Global Journal of Health Science. 2016; 8(11): 62-68.

20. Poorgholami F, Javadpour SH, Saadatmand V, KargarJahromi M. Effectiveness of Self-Care Education on the Enhancement of the SelfEsteem of Patients Undergoing Hemodialysis. Global Journal of Health Science. 2016; 8(2): 132-136.

21. KargarJahromi M, Javadpour SH, Taheri L, Poorgholami F. Effect of Nurse-Led Telephone Follow ups (Tele-Nursing) on Depression, Anxiety and Stress in Hemodialysis Patients. Global Journal of Health Science. 2016; 8(3): 168-173.

22. Jahromi M. K, Poorgholami F, Rahmanian F, Rahmanian E. Effects of Self-Care Education with Telephone Follow-up on Self-Efficacy level in Hemodialysis Patients. Biosci Biotechnol Res Asia 2016; 13(1) 